\title{
The Experience of Social Touch in Multi-User Virtual Reality
}

\author{
Philipp Sykownik \\ philipp.sykownik@uni-due.de \\ University Duisburg-Essen \\ Duisburg, Germany
}

\author{
Maic Masuch \\ maic.masuch@uni-due.de \\ University Duisburg-Essen \\ Duisburg, Germany
}

low intimacy body contact

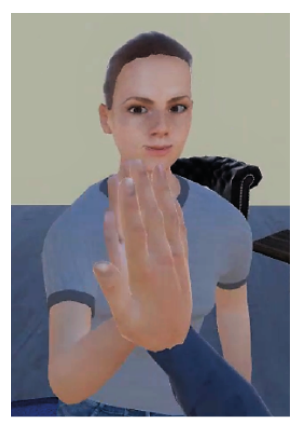

high-five

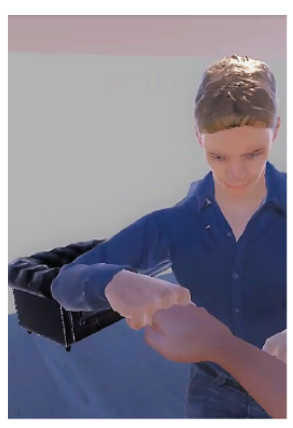

fist-bump

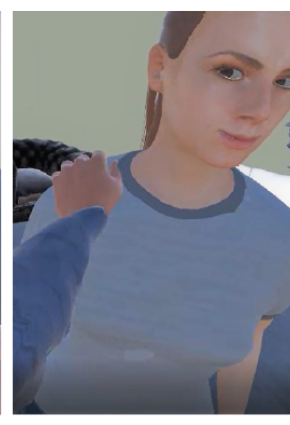

shoulder tap high intimacy body contact

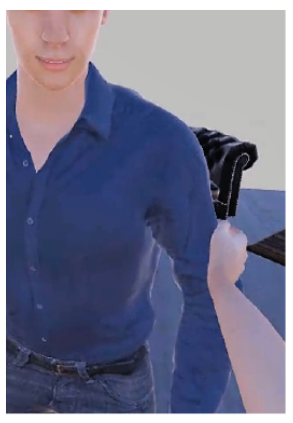

caress arm

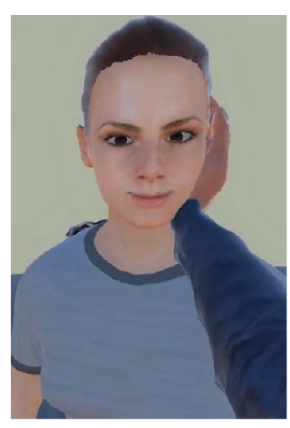

caress face

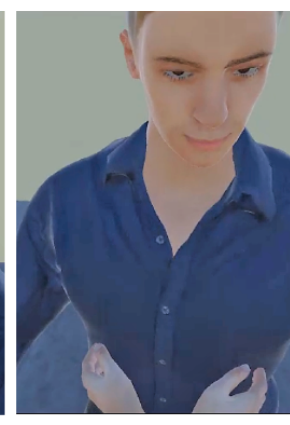

hug

Figure 1: Participants performed the interactions depicted above with a physically remote research assistant in VR. We compared the emotional experience of the two intimacy groups (high vs. low intimacy) regarding the influence of intimacy, direction of touch and sex of participant.

\begin{abstract}
We present user study results on virtual body contact experience in a two-user VR scenario, in which participants performed different touches with a research assistant. The interaction evoked different emotional reactions in perceived relaxation, happiness, desire, anxiety, disgust, and fear. Congruent to physical social touch, the evaluation of virtual body contact was modulated by intimacy, touch direction, and sex. Further, individual comfort with interpersonal touch was positively associated with perceived relaxation and happiness. We discuss the results regarding implications for follow-up studies and infer implications for the use of social touch in social VR applications.
\end{abstract}

\section{CCS CONCEPTS}

- Human-centered computing $\rightarrow$ Empirical studies in $\mathrm{HCI}$; Virtual reality; Laboratory experiments.

\section{KEYWORDS}

social VR, multi-user VR, virtual reality, social touch, mediated social touch

\section{(1)}

This work is licensed under a Creative Commons Attribution-NonCommercial-NoDerivs International 4.0 License.

VRST '20, November 1-4, 2020, Virtual Event, Canada

(C) 2020 Copyright held by the owner/author(s).

ACM ISBN 978-1-4503-7619-8/20/11.

https://doi.org/10.1145/3385956.3418944

\section{ACM Reference Format:}

Philipp Sykownik and Maic Masuch. 2020. The Experience of Social Touch in Multi-User Virtual Reality. In 26th ACM Symposium on Virtual Reality Software and Technology (VRST '20), November 1-4, 2020, Virtual Event, Canada. ACM, New York, NY, USA, 11 pages. https://doi.org/10.1145/3385956.3418944

\section{INTRODUCTION}

Given the advance of current VR hardware and ambitions in developing social VR environments such as Facebook Horizon, embodied social interactions within virtual worlds are now more accessible than ever. For instance, virtual social touch is already used as a social feature that adds to the overall user experience (e.g., a handshake for friending in RecRoom). Unfortunately, the freedom of embodied social interaction like virtual social touch seems not to be limited to positive experiences, as physical harassment (e.g., unwanted "touching" of avatars) is already occurring in social VR applications [5]. However, based on a current taxonomy for the design of social VR research and industry applications, social touch does not yet seem to have received significant attention in studies on social VR [29]. Although the taxonomy identifies physical expressions from the body of literature as a communication type of interest in current VR prototypes, the work cited in the taxonomy does not yet investigate this communication type as an independent feature. Further, a recent overview of nonverbal communication channels utilized by current social VR platforms also indicates that social touch is not yet considered as a core feature [56]. However, as interpersonal touch is so vital for human interaction in the physical world [12, 30, 47, 52], we do not see any reason to believe that it should not become a central aspect of virtual social interaction in the future. Therefore, we investigate the emotional reactions to virtual body contact to aid the design of virtual social interactions. 


\section{SOCIAL TOUCH}

Humans have a natural need for social physical contact, and touching and being touched are evolutionary established basic modes for interaction $[8,12]$. Early observational and more recent self-report studies indicate that the use and subjective evaluation of interpersonal touch in the real world underlies complex interactions of influencing factors like the social context, sex, direction of touch, the type and location of touch, and the type of relationship between interactors [15, 19, 24, 46, 53, 54].

Interpersonal touch is one of the primary means to foster and express intimacy between each other [24,57], and different types and locations of touch are associated with varying degrees of intimacy $[24,30]$. Generally, areas, where touch is perceived as less intimate are the hands, arms, and shoulders. These areas are allowed to be touched by strangers or emotionally distant acquaintances. Moderate intimacy areas include the face, stomach, chest, buttock, and the back of thighs, which are at most accepted to be touched by close friends, family members, or the partner. High intimacy areas are the thighs' insides and pubic areas and only accepted to be touched by the romantic partner. This classification is more or less consistently reported in the literature. However, it can differ in varying degree depending on the direction of touch (initiator and receiver), sex of touch initiator and receiver, their cultural background, and emotional bonding $[25,54,58]$, as well as their sexual orientation [14], and individual traits like social anxiety [31, 51].

Among the various influencing factors, sex is one of the most often studied moderators on the effects of touch [24], and a recent meta-review of work concerned with the neurophysiology of affective touch supports the early indications regarding a moderating effect of sex on social touch perception [46]. Whereas for heterosexual women, in particular, a touch must reflect the relationship's intimacy to be appropriate and pleasant, heterosexual men tend to perceive intimate touches from stranger women as pleasant [25]. Generally, heterosexual women and men tend to prefer touches from women over touches from men [53, 54, 58]. Compared to different-sex touches, same-sex touch among heterosexual strangers was found to be often rated as unpleasant or inappropriate, but women tend to be more open to it than men $[13,25,54]$.

\section{MEDIATED SOCIAL TOUCH IN VR}

Modern VR technology can induce a strong illusion of virtual body ownership or a sense of embodiment [28, 34, 40, 43, 49,50]. As a consequence of perceiving an illusion of virtual body ownership, a user may experience events affecting his virtual body, as if they would affect his physical body. Thus, observing one's virtual body being touched by or touching another avatar could induce similar reactions as being touched in the real world. Indeed, technologically mediated social touch seems to evoke physiological, emotional, and behavioral reactions similar to its real-world counterpart [18, 27, $60,60]$. These findings mainly stem from the evaluation of haptic feedback devices that often transmit haptic stimuli independent from the visual presentation of the interaction $[1,18]$. However, the development of such interfaces, in particular, to enable realistic haptic interaction in consumer VR, is an ongoing research area, that does not seem to evolve into affordable consumer-level devices soon
$[3,9,38]$. Though, research on cross-modal sensory interactions indicates that visual cues alone seem to sufficiently induce reactions similar to being touched $[4,6,41]$. And as modern consumer VR technology is already a remarkable visual medium, we highlight the importance to understand the reactions to mediated social body contact that is primarily induced by visual cues generated by such hardware.

Findings from studies that used VR technology are based on diverse hardware and software configurations, sometimes limited by the technology available at that time. However, they can be classified according to the interaction partners (human-human vs. human-agent) and the direction of the touch investigated (participant initiates vs. receives touch).

One of the most recent studies compared human-agent and human-human interaction in VR were participants received a touch on their physical shoulder. The results indicate that incorporating social touch in the virtual interaction could increase the touching virtual agent's perceived humanness but not of the humancontrolled avatar's[26]. Another recent study (human-agent, receiving touch) found that the perceived appropriateness and erogeneity of virtual touch on different body zones of an embodied avatar seem to be modulated by the same influencing factors as physical touch (i.e., the location of touch on the virtual body, the sex of the touching avatar and the touched participant, and sexual orientation). The touches were induced solely by visual stimuli [14]. Another relatively recent study (human-agent, receiving and initiating) that offered low immersive fidelity compared to the two prior studies investigated the role of touch in economic decision making [55]. Contradicting to previous findings in VR settings (human-agent, receiving touch) [21,65], the authors found no effect of touch on compliance behavior [55],

In a study on how anti-fat attitudes affect touching behavior with force-feedback devices in VR (human-agent, initiating) touch duration and strength were affected by factors like sex, anti-fat attitudes, and the virtual agent's characteristics, similar to face-to-face studies from that field [59]. Another study from that time (humanagent, receiving) found that virtual touch perception supported by tactile feedback seems to be modulated by facial expressions of the touching and individual differences related to participants' sex [20].

Seemingly the only prior study that investigated the effects of virtual touch in a multi-user VR scenario investigated the role of physical feedback to a received touch on one's virtual shoulder on the tendency to engage in an embarrassing social situation (i.e., sing in front other avatars) [7]. Tactile feedback to the touch did not indicate increased the tendency to sing, but the perceived realism of the touch [7]. An early study (human-agent, initiating touch) found that virtual agents tend to be touched with less force than virtual geometric objects using a force-feedback input device. Further, the force used on virtual agents depended on the location of the touch and the sex of the agent [2].

This short review illustrates that previous studies have rarely investigated direct emotional responses to virtual touch. If, only in limited ways and not in terms of several distinct emotions [14, 26]. Most studies have been interested in the haptic experience of touch $[7,20]$, touching behavior, and behavioral responses [2, 7, 55, 59, 65]. 
Table 1: Application Structure

\begin{tabular}{cl}
\hline Scene & Purpose \\
\hline Tutorial & $\begin{array}{l}\text { Avatar customization: leg and arm length, sex, } \\
\text { hair, skin tone, eyes. Virtual mirror. Dummy } \\
\text { model to practice touches. }\end{array}$ \\
1st Interaction & $\begin{array}{l}\text { Each user initiates and receives several social } \\
\text { body contacts of intimacy group 1. }\end{array}$ \\
Pause & $\begin{array}{l}\text { Users wait in separated virtual rooms in front } \\
\text { of a mirror before entering the second inter- } \\
\text { action scene. } \\
\text { 2nd Interaction }\end{array}$ \\
& $\begin{array}{l}\text { Each user initiates and receives several social } \\
\text { body contacts of intimacy group 2. }\end{array}$ \\
\hline
\end{tabular}

Further, in particular, older but also some recent studies used hardware and software configurations that only provided limited immersive characteristics compared to today's available hardware (e.g., no virtual user representation [26, 55], no full-body movement [14]). Thus, we consider investigating the affective reactions to virtual touch in terms of distinct emotions in a multi-user VR scenario that resembles the immersive quality of current consumer social VR applications as a valuable contribution to this research field.

\section{APPLICATION DESCRIPTION}

We developed a two-user VR application that requires users to perform various social body contacts while providing full-body user representations and movement tracking (see Figure 1). Table 1 provides a quick overview of the application structure.

Both users go through a tutorial scene separated from each other to take enough time to prepare for the actual interaction. The tutorial scene has three functions. The first is to familiarize users with the virtual environment and hardware. Further, their avatars' individualization in front of a virtual mirror should promote presence and embodiment [61]. Finally, users can become familiar with the execution of virtual body contact by practicing the social touch interactions on a static dummy avatar model.

We designed the two interaction scenes as a series of simple interaction tasks, where users have to perform different social touch interactions from one of the two groups that Figure 1 illustrates. The interaction takes place in a virtual living room where a monitor displays the interactions to perform. The application recognizes correctly performed interactions, plays a confirmation sound, and updates the virtual monitor's instructions correspondingly. For interactions with an initiator and receiver role, the system indicates who should initiate the interaction (e.g., A hugs B). In sum, the application requires each user to perform the following interactions:

- low intimacy body contacts: $8 \mathrm{x}$ high-five, $8 \mathrm{x}$ fist-bump, $4 \mathrm{x}$ initiating shoulder tap, $4 \mathrm{x}$ receiving shoulder tap

- high intimacy body contacts: $5 \mathrm{x}$ initiating caress arm, $5 \mathrm{x}$ receiving caress arm, $5 x$ initiating caress face, $5 x$ receiving caress face, $5 \mathrm{x}$ initiating a hug, $5 \mathrm{x}$ receiving a hug

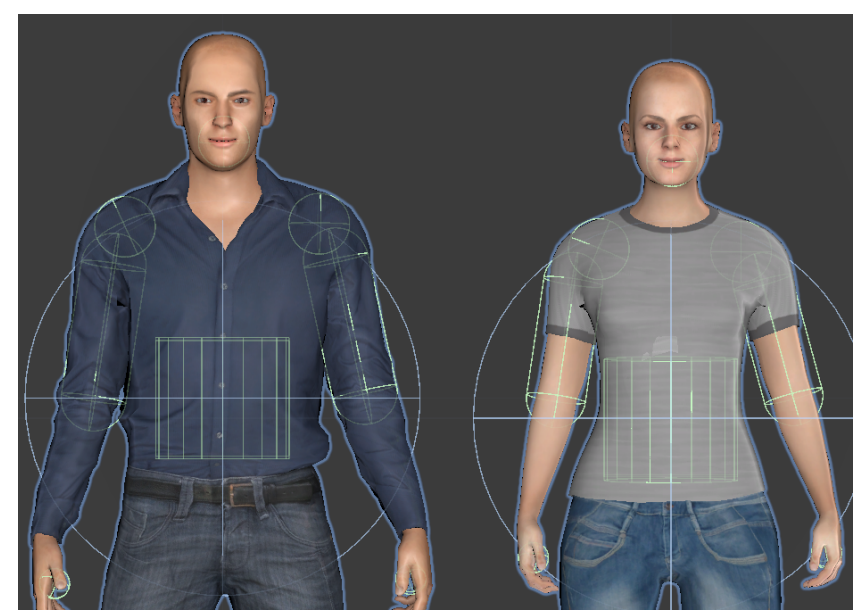

Figure 2: Avatar base models with colliders that trigger pseudo-haptic effects on collision with virtual hands: partner's hands, head, shoulders, upper arms, and abdomen.

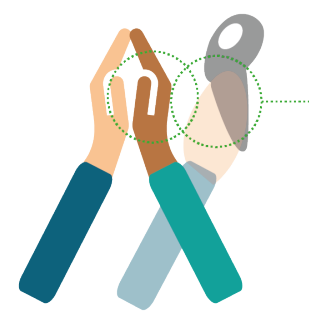

a) virtual hand does not permeate certain other body parts

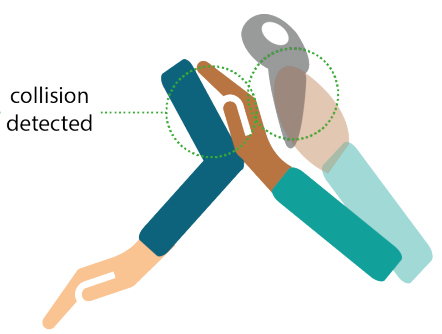

b) collision of the virtual hand with near body parts is maintained
Figure 3: Our pseudo-haptics approach: a user's virtual hand is decoupled from a trigger object that follows the user's physical hand's movement. This decoupling is triggered when the collider attached to the trigger object collides with colliders of certain target body areas.

A pause scene separates the two interaction scenes. There, users can get used to the VR setting again after filling out desktop-based questionnaires in a study setting.

\subsection{Selection of Social Touches}

Figure 1 presents the six social touch-types we used to induce low and high intimacy during the interactions. High-five, fist-bump, and shoulder patting are interactions related to body regions and tactile stimuli associated with low intimacy [25, 58]. Caressing the arm and face, or touching the torso (hugging) are associated with high intimacy $[25,58]$.

\subsection{Avatar Collision}

To prevent users from permeating each other's avatar and thus increasing realism, we used pseudo-haptic effects. Pseudo-haptics aim to simulate haptic perceptions using visual cues [36, 37], usually realized by creating a positional offset between the virtual and 
physical hand and have recently found to positively affect the immersive experience and enjoyment during interaction with virtual objects [44].

In our application, we added colliders to specific body areas of the avatar models (Figure 2). Thereby, the colliders associated with the avatar hands serve a particular function, as these are not attached to the virtual hands but an empty object that, in turn, follows the respective physical hands' movements. During the interaction, each avatar hand follows this object. When it detects a collision with a specific body part (e.g., the other user's upper arm), the associated hand model is attached to that location with a predefined pose. At the same time, the empty object keeps following the user's physical hand. The virtual hand, however, remains attached, as long as the hand collider detects a collision with the target area (Figure 3). Further, touching the other avatar triggers vibrotactile feedback from the Vive controller.

\subsection{Apparatus}

The application was developed with the Unity game engine and tested with wireless HTC Vive and Vive Pro headsets. The avatars were created using the MakeHuman software and animated based on tracking information and inverse kinematics using the Final IK library for Unity. For simulating eye gaze behavior, we used the Simple Eye Gaze plugin for Unity. The networking components of the application were implemented using the Mirror Networking API. Voice Chat is not supported. User movement is tracked with the VR headset, two Vive controllers, and two Vive trackers mounted to the feet. Virtual hand postures can be adapted by pressing the grip button for a flat hand or trigger button for making a fist.

\section{USER STUDY}

We contribute to the research on social VR and mediated social touch by evaluating the experience induced by virtual social touches of different levels of intimacy performed with a stranger. This scenario should reflect the social interactions in current social VR applications where users can interact with strangers worldwide. We evaluated the affective response in terms of several emotions of different valence. The stranger in our study was a remote research assistant to assert some degree of standardization. To control the potential influence of sexual orientation, we only recruited heterosexual participants that we required to interact with an avatar of the opposite sex. We let participants receive and initiate virtual social touches of different degrees of intimacy, to investigate the interrelationship between the direction of touch with intimacy and participants' sex. As we focus on the impact of intimacy, we subdivided only the intimacy groups into two separate interaction scenes, while integrating the receiving and initiating role in each of these two scenes. The alternative would have been to create four conditions. This design would have required participants to leave and enter VR three times between the conditions in contrast to only one time between the two conditions we have used.

\subsection{Participant Information}

We conducted a within-subject user study with 44 participants living in Germany (27 female) aged from 18 to $31(M=23, S D=$ 3.4). Participants customized their avatars to reflect their physical appearance (e.g., 24 participants selected the white, 16 the slightly tanned, three the tanned, and one the black skin color for their avatar). Most participants had few prior VR experiences: 2 use VR more than once a month; 13 indicated to use VR occasionally; 15 used VR once before; 14 never used VR before. Only two participants had prior experience with social VR applications. We recruited participants mainly at the university and via social network sites. Forty-one participants were students. Some students were required to collect hourly credits for passing a specific study course module and were compensated correspondingly (1.5 hours). The faculty's ethics committee approved the study.

\subsection{Procedure}

Participants were welcomed and informed about the course of the study. They were told that they had to interact in VR with another participant who was unknown to them and in another physical space. The male and female avatar variants of the researchers had the same visual appearance in each study run (see Figure 1). After giving their consent, participants were asked to complete a desktop-based questionnaire on demographic data. They were then familiarized and equipped with the VR equipment. Audio instructions guided the participants through the application. Participants were asked to make their avatar resemble their physical appearance by using pre-defined assets (i.e., the color of skin, eyes, and hair, hairstyle). After the tutorial, participants completed the two interaction scenes in randomized order. VR exposure lasted from 20 to 35 minutes. Eventually, we debriefed participants and informed them that they interacted with a research assistant. After each interaction scene, participants completed desktop-based questionnaires assessing their experience.

\subsection{Measures}

5.3.1 Individual Factors. We measured the comfort with receiving and initiating interpersonal touch of the participants to evaluate if this real-world trait is associated with the experience of virtual social body contact. As a measure, we applied the Comfort with Interpersonal Touch (CIT) scale that requires to rate 6 items on 7-point likert-scale "strongly disagree" - "strongly agree"[62].

5.3.2 Immersive Experience. To control if the application offered a comparable immersive quality in both conditions (low and high intimacy), we assessed perceived spatial presence, aspects of virtual body ownership, and co-presence.

We applied the spatial presence subscale of the iGroup Presence Questionnaire (IPQ) [48]. Items were rated on a scale from 1-7 where higher scores indicate a higher presence. Four item scales ranged from "fully disagree" - "fully agree." One item scale ranged from "did not felt present" - "felt present."

To assess the possible sensation of embodiment based on movement tracking and avatar animation in our two conditions, we selected three items associated with body ownership and agency from an item pool used to construct an embodiment questionnaire [16] (items presented in Table 2). We selected only these three items as we considered them to be sufficient in terms of our analysis goal for the embodiment-related immersive experience. We thus did not intend to use them as a valid instrument to assess the extensive concept of embodiment but to analyze them on a per item basis. 
Each item was rated on a scale ranging from 1-7 ("strongly disagree" - "strongly agree").

We applied the co-presence subscale of the Networked Minds Measure of Social Presence (NMMSP) [23]. This scale includes six items that allow us to rate how far our application generated a social situation with mutual attention and action-taking. Items were rated on a scale ranging from 1-7 ("strongly disagree" - "strongly agree").

We further applied the Simulator Sickness Questionnaoire (SSQ) to measure the perceived simulator sickness [33]. Spacial presence, embodiment, and co-presence were assessed after each interaction scene. The SSQ was applied at the end of the experiment.

5.3.3 Emotional Experience. We applied the Discrete Emotions Questionnaire (DEQ) [22] to assess the emotional response to the virtual touches in terms of experienced relaxation, happiness, desire, anxiety, disgust, and fear. The DEQ measures the intensity of several experienced emotions. It assesses each discrete emotion with four individual items (e.g., happiness: happy, satisfaction, enjoyment, liking). Participants had to indicate to what extent they experienced the corresponding feeling after each interaction scene, separated for initiated and received touches (scale from 1-7, "not at all"-"to some extent"-"extremely").

5.3.4 Internal Consistency of Measures. Most subscales yielded consistently acceptable $\alpha$ values greater than .66 or .80 (i.e., CIT, SSQ, NMMSP, Relaxation, Happiness, Anxiety, Disgust, Fear $)^{1}$. The embodiment items' internal consistency was questionable when combined into one subscale (between .50 and .60). Nevertheless, as already stated, we intended to analyze the items on a per question basis. Cronbach's $\alpha$ values for desire were not consistently acceptable, as in two conditions, the value was between .50 and .60 . We excluded the item "wanting" from this scale, resulting in $\alpha$ values above .8 in each condition.

\section{RESULTS}

First, we present the results of the analysis of the immersive experience. Second, we present the emotional experience's descriptive results, followed by an inferential analysis of the assumed effects of intimacy, touch direction, and sex. Third, we present results regarding the association between comfort with interpersonal touch and the emotional experience of received and initiated touch. All significance tests were conducted on a .05 significance level.

\subsection{Immersive Experience}

The high and low intimacy interaction scenes induced moderate to high perception of spatial presence and co-presence (Table 2). Two Wilcoxon signed-rank tests were calculated due to rejected normality assumption of the difference scores. The test did not reveal a significant difference in terms of spatial presence $(Z=$ -.698, $p=0.485)$ or co-presence $(Z=-1.662, p=.096)$.

On average, participants had a moderate perception that the avatar was their own body and felt that they were controlling it as it was their own. Correspondingly, they seem not to have had the feeling, that it was moving on its own. Due to rejected normality assumption of the difference scores, as well as to non-symmetric score distributions we calculated three exact sign tests that did not

\footnotetext{
${ }^{1}$ Please refer to the auxiliary material for exact $\alpha$ values for each subscale per condition
}

Table 2: Immersive experience during low and high intimacy interactions assessed on 7-point likert-scales.

\begin{tabular}{lcc}
\hline Measure & $\mathrm{M}(\mathrm{SD})$ low & $\mathrm{M}(\mathrm{SD})$ high \\
\hline $\begin{array}{l}\text { Presence } \\
\text { Spatial Presence }\end{array}$ & $5.49(.864)$ & $5.41(.892)$ \\
$\begin{array}{l}\text { Embodiment } \\
\text { It felt like the avatar } \\
\text { was my own body. }\end{array}$ & $4.2(1.59)$ & $4.43(1.59)$ \\
$\begin{array}{l}\text { It felt like I could } \\
\text { control it as if it was } \\
\text { my own body. }\end{array}$ & $5.34(.963)$ & $5.25(.811)$ \\
$\begin{array}{l}\text { It felt like it was moving } \\
\text { on its own. }\end{array}$ & $1.18(1.06)$ & $1.25(1.08)$ \\
$\begin{array}{l}\text { Social Presence } \\
\text { Co-Presence }\end{array}$ & $5.47(1.04)$ & $5.71(.951)$ \\
\hline
\end{tabular}

reveal a significant difference of median scores of the items between the two conditions: "my own body" exact binomial $p$ (2-tailed $)=$ 0.405 , "control" exact binomial $p(2$-tailed $)=0.454$, "on its own" exact binomial $p$ (2-tailed $)=0.481$.

No participant wanted to stop the experiment or mentioned impairments of well-being due to VR exposure. The mean total SSQ score is relatively low $31.79(S D=26.51)$, with 235.62 being the possible maximum score.

In conclusion, we retained the assumption that both conditions provided the same immersive experience and thus did not consider the immersive experience in the following analysis.

\subsection{Emotional Experience}

Figure 4 illustrates the emotional experience based on the DEQ scores. Mean and median values indicate that participants felt relaxation and happiness with moderate intensity, slight feelings of anxiety, and little to no desire, disgust, or fear. Thus, most participants' perceived emotional reaction was moderately intense at most and somewhat of positive valence. However, the individual observations illustrated in Figure 4 indicate partly substantial inter-individual differences between participants' emotional experiences. Reported relaxation and happiness scores range between low and very high values independent from intimacy or the direction of touch. Further, some participants experienced moderate anxiety levels during low intimacy touches, and high degrees of anxiety during high intimacy touches, independent from touch direction. Also, for the emotions desire, disgust, and fear, there are inter-individual differences with observations in the range of no to moderate intensity.

6.2.1 Inferential Analysis. Relaxation and happiness data met assumptions for parametric testing. Thus we conducted two 2(intimacy) $\mathrm{x} 2$ (direction) $\mathrm{x} 2$ (sex) repeated measures ANOVAs on the relaxation and happiness scores with intimacy and direction as within-subject factors and sex as a between-subject factor. Desire, anxiety, disgust, and fear data did not meet requirements for parametric testing (i.e., normality of residuals, symmetric distributions, no extreme outliers). Thus, we conducted a non-parametric factorial analysis of repeated measures data by using the f1.ld.f2 function 

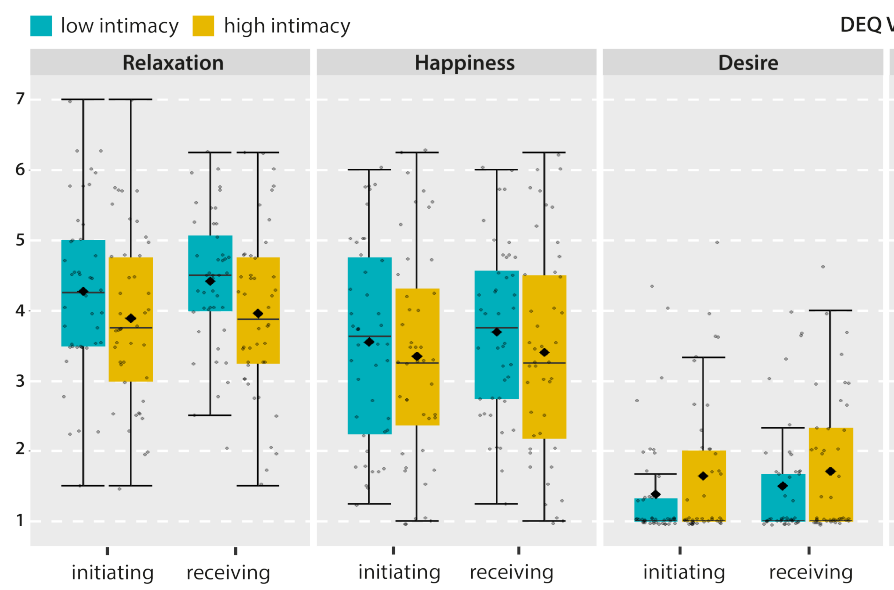

DEQ Values
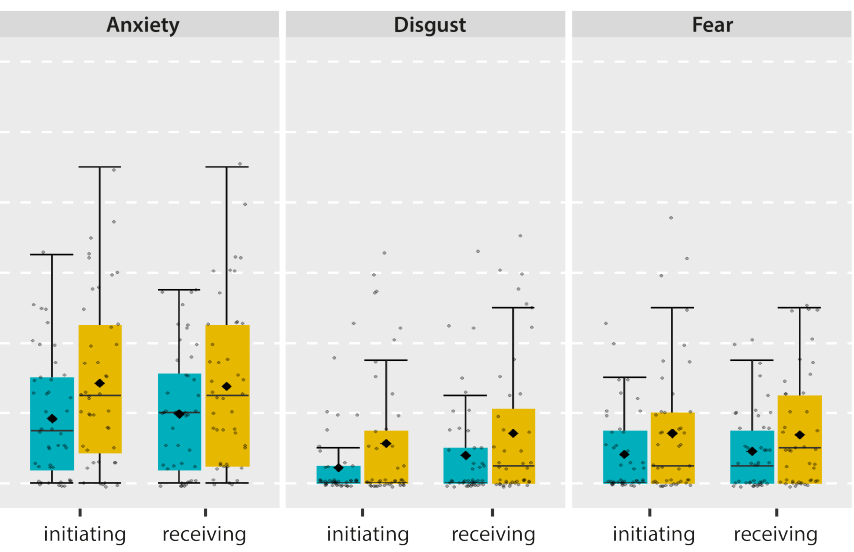

Figure 4: Boxplots with median, mean (diamond), interquartile distance (box), range (whiskers, excluding outliers) and individual observation values (gray points) for the DEQ subscales grouped by intimacy and touch direction. Assessed on 7-point likert-scale: 1 (not at all) - 4(some extent) - 7(extremely)

Table 3: Main and interaction effects of intimacy, touch direction, and sex on DEQ scores.

\begin{tabular}{|c|c|c|c|c|c|c|c|}
\hline ANOVA & Relaxation & Happiness & npar ANOVA & Desire & Anxiety & Disgust & Fear \\
\hline Sex & & & Sex & & & & \\
\hline$F(1,42)$ & 4.98 & 1.85 & $F(1, \infty)$ & 5.38 & 3.54 & 2.48 & 7.08 \\
\hline$p$ & .031 & .182 & $p$ & .020 & .060 & .115 & .007 \\
\hline$\omega \hat{2}$ & .044 & .010 & & & & & \\
\hline Intimacy & & & Intimacy & & & & \\
\hline$F(1,42)$ & 9.37 & 6.57 & $F(1, \infty)$ & 6.66 & 8.54 & 9.06 & 3.82 \\
\hline$p$ & .004 & .014 & $p$ & .001 & .003 & .003 & .051 \\
\hline$\omega \hat{2}$ & .036 & .006 & & & & & \\
\hline Direction & & & Direction & & & & \\
\hline$F(1,42)$ & 1.45 & 5.12 & $F(1, \infty)$ & 3.47 & .040 & 11.3 & 2.10 \\
\hline$p$ & .235 & .029 & $p$ & .062 & .842 & $<.001$ & .148 \\
\hline$\omega \hat{2}$ & .000 & .001 & & & & & \\
\hline Sex $x$ Int & & & Sex $x$ Int & & & & \\
\hline$F(1,42)$ & .208 & .492 & $F(1, \infty)$ & 7.28 & .311 & 2.06 & .157 \\
\hline$p$ & .651 & .487 & $p$ & .007 & .577 & .151 & .693 \\
\hline$\omega \hat{2}$ & .000 & .000 & & & & & \\
\hline Int $x$ Dir & & & Int $x$ Dir & & & & \\
\hline$F(1,42)$ & .209 & .245 & $F(1, \infty)$ & .006 & .283 & .664 & .575 \\
\hline$p$ & .650 & .623 & $\mathrm{p}$ & .936 & .594 & .415 & .448 \\
\hline$\omega \hat{2}$ & .000 & .000 & & & & & \\
\hline Sex x Dir & & & Sex x Dir & & & & \\
\hline$F(1,42)$ & .111 & 3.03 & $F(1, \infty)$ & .670 & .200 & .251 & .238 \\
\hline$p$ & .741 & .089 & $p$ & .413 & .654 & .616 & .625 \\
\hline$\omega \hat{2}$ & .000 & .000 & & & & & \\
\hline Sex $x$ Int $x$ Dir & & & Sex x Int x Dir & & & & \\
\hline$F(1,42)$ & .010 & 3.82 & $F(1, \infty)$ & .683 & 4.01 & 11.4 & .054 \\
\hline$p$ & .919 & .057 & $p$ & .408 & .045 & $<.001$ & .816 \\
\hline$\omega \hat{2}$ & .000 & .000 & & & & & \\
\hline
\end{tabular}




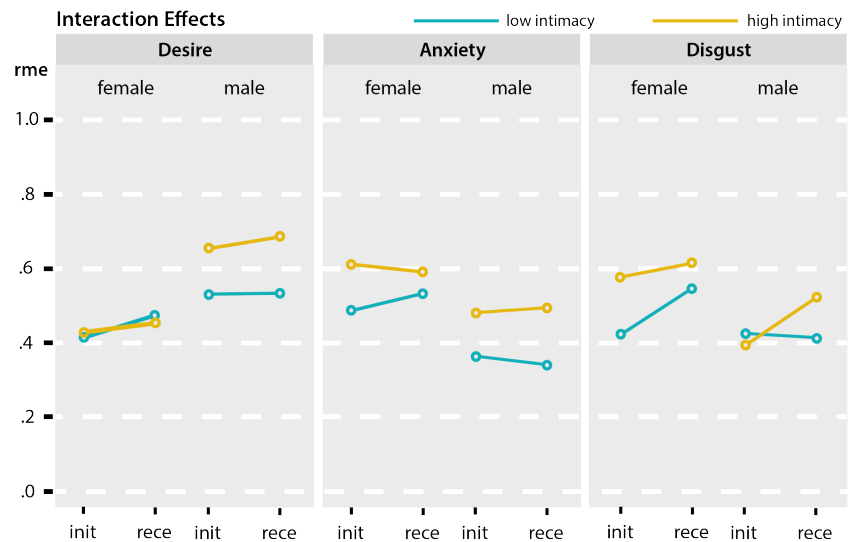

Figure 5: Profile plots of the relative marginal effects (rme) showing the detected interaction effects: Sex $x$ Direction on desire, Sex $x$ Intimacy $x$ Direction on Anxiety and Disgust

from the $n$ parLD R package ${ }^{2}$ to test for main and interaction effects $[11,42]$.

6.2.2 Influence of Intimacy, Involvement \& Sex. We found significant main effects for sex and intimacy on relaxation, intimacy and direction of touch on happiness, and sex on fear. Results are summarized in Table 3. Further, we found a second-order interaction effect of sex and intimacy on desire and third-order interaction effects of sex, intimacy, and direction of touch on anxiety and disgust. We did not further decompose detected second- and third-order interactions effects as this process would have required an extensive report of results. Alternatively, Figure 5 provides an intuitive assessment of how the individual factors have interacted. The non-parametric factorial analysis calculates the rme values (relative marginal effect) provided in Figure 5. They are interpreted as the probability that a randomly chosen observation from the whole dataset, has a smaller value than a randomly chosen observation from the subgroup of interest. Thus, it corresponds with the average scores reported by a subgroup (e.g., $r m e_{\text {fear,male }}=.387$ means: with an estimated probability of .387 a fear score randomly chosen from the whole dataset is smaller than a fear score randomly chosen from the scores reported by men)[42].

6.2.3 Relaxation. We found significant main effects for sex and intimacy on reported relaxation. Male participants were more relaxed $(M=4.54, S D=1.16)$ than female participants $(M=3.88, S D=$ 1.09). However, participants reported higher intensity of relaxation for low intimacy touches $(M=4.35, S D=1.09)$ than they did for high intimacy touches $(M=3.95, S D=1.20)$. The effect sizes are small to medium $(.01<\omega \hat{2}<.06)$.

6.2.4 Happiness. We found significant main effects for touch direction and intimacy on reported happiness. Participants reported more happiness when they were touched $(M=3.55, S D=1.39)$ than they did when they touched their partner $(M=3.45, S D=1.46)$. Participants reported higher scores of happiness for low intimacy

\footnotetext{
${ }^{2}$ https://rdrr.io/cran/nparLD/man/f1.ld.f1.html last access 20th of August 2020
}

touches $(M=3.63, S D=1.31)$ than for high intimacy touches $(M=$ $3.38, S D=1.53)$. The effect sizes are small $(\omega \hat{2}<.01)$.

6.2.5 Desire. We found a second-order interaction effect of sex and intimacy on the perceived desire. Compared to women, men's perception of desire intensified with higher intimacy of touches. And women tended to report generally less desire than men (Figure $5)$.

6.2.6 Anxiety. We found a third-order interaction effect of sex, intimacy, and direction on the perceived anxiety. On average, men tended to report less anxiety than women. Thereby, for men and women, high intimacy touches induced more anxiety than low intimacy touches. However, for women, this effect of intimacy depended on the direction of touch. They felt more anxiety when they received low intimacy touches than when they initiated low intimacy touches. In contrast, their anxiety scores for received touches of high and low intimacy did differ only slightly. A slightly inverse interaction of intimacy and direction applies to men (Figure fig:profile).

6.2.7 Disgust. There was a third-order interaction effect of sex, intimacy, and direction on the perceived disgust. The sensation of disgust depended very much on the specific constellation of the three factors. Women reported more disgust while initiating intimate touches than for initiating low intimacy touches. For men, there was no difference between the two levels of intimacy for initiated touches. On the other hand, for women, intimacy seemed to make little difference when they were touched, as anxiety only differed slightly between high and low intimacy touches they received. For men, on the other hand, the sensation of disgust was more intensive while being touched with high intimacy (Figure 5).

6.2.8 Fear. There was a significant main effect for sex on reported fear. In general, female participants reported a greater amount of fear (Mean Rank $=100.97, r m e=.571)$ than did male participants $($ Mean Rank $=68.69 \mathrm{rme}=.387)$.

6.2.9 Comfort with Interpersonal Touch. We found positive associations between comfort with interpersonal touch and the happiness and relaxation experienced during intimate touches. Comfort with initiating touch was found to be strongly positively correlated with relaxation $(r(42)=.502, p<.001)$ and moderately with happiness induced by initiated intimate touches $(r(42)=.349, p=.020)$. Comfort with receiving touch was found to be strongly positively correlated with relaxation $(r(42)=.516, p<.001)$ and moderately with happiness induced by received intimate touches $(r(42)=.386, p=$ $.010)$.

\section{DISCUSSION}

Our results show that virtual body contact can be a diverse emotional experience, and depending on individual differences, it can be quite intense or relatively shallow. Moreover, our inferential analysis revealed that the affective reaction was modulated by factors that are known to modulate unmediated social touch (i.e. intimacy, participants' sex, and the direction of touch). Further, the intensity of positive reactions was associated with the general individual comfort with interpersonal touch. In particular, intimacy and participants' sex modulated the emotional reaction. 
In sum, the increased intimacy was associated with less favorable (relaxation, happiness) and stronger adverse reactions (anxiety, disgust, fear). Women tended to be stronger affected by this effect, partially also depending on whether they touched their partner or were touched by him. Interestingly, although men and women perceived only slight feelings of desire for low intimacy touches, men reacted to the intimate touches with an increase in perceived desire, whereas women did not. Our findings align with how social touch of varying intimacy between heterosexual opposite-sex strangers is perceived in the real world. That is, for women a touch must reflect the intimacy of the relationship to be appropriate and pleasant, whereas men are inclined to be more willing to tolerate intimate interaction with a stranger women $[25,54,58]$.

Beyond contributing to research on mediated social touch by confirming previous work that virtual body contact can evoke reactions similar to its real-world counterpart, we contribute to this field in several ways. We assessed the affective reaction in terms of six different emotions, thus providing a nuanced look on different facets of the user experience beyond measures used in prior studies like pleasantness, appropriateness, erogeinity, and compliance $[7,14]$. Thereby, our results show that virtual social touch can simultaneously evoke multiple emotions of varying valence. By investigating initiated and received touches in a single study, we acknowledged the bidirectional nature of social touch. Consequently, we identified interrelationships of touch direction with intimacy and sex [24].

Concerning older prior work, we contribute to the field by using a VR setup that equals consumer-level scenarios that, compared to other recent studies, utilized full-body motion tracking and userrepresentation in the context of virtual social touch. Further, our and recent related results [14] highlight, that it is also imperative to investigate mediated social body contact that is primarily induced by visual cues and does not rely on sophisticated haptic feedback devices.

Further, our results demonstrate that even in a prescribed, neutral situation, without provoking visual stimuli (like an avatar in underwear [14]), virtual social touch can, although with limited intensity, induce diverse emotional reactions.

Furthermore, our results inform previous studies on perceived humanness of agents, by motivating follow up studies to consider intimacy of touch as a parameter to increase perceived agent and avatar humanness [26] further.

We admit that the effects we detected and the reported intensities of emotional experiences are small and consider the study setting's following characteristics as a potential explanation. Our study scenario differed from the real world as participants could not speak with their interaction partners. Further, the avatars did not react with non-verbal social signals like changes in mimic to the touches, that could have affected emotional responses [10, 20]. However, by controlling these factors and controlling the course of the interaction by a prescribed procedure, our experimental scenario lacked the spontaneous nature of real-world social interactions. Correspondingly, we assume that the limited available communication channels and the prescribed course of interaction may have limited the intensities of emotional reactions and the effects of the identified influencing factors. On the other hand, this interpretation leads to the assumption that in a spontaneous virtual social interaction, in which the users may be more personally involved, the emotional reactions may be of higher intensity.

Moreover, as we applied subjective measures in retrospect, it is, of course, possible that the participants' spontaneous emotional reaction intensity was no longer salient. Also, the majority of participants had few or no prior VR experiences. As participants may have been overwhelmed by the novel experience in general, a potentially more substantial effect of the touches could have been masked.

\subsection{Limitations}

Our results have to be interpreted with the following limitations in mind. We have combined three forms of interaction in one of two groups and have not evaluated them individually. Of course, this means that information was lost. Perhaps the individual interactions within each group could have had opposing effects on the experience based on the degree of intimacy the participants associated with the individual touches.

The emotional evaluation of the received touches could have been confounded by the touch's limited visual stimuli (due to the headset's limited field of view) [32]. In particular, during the hug interaction, we assume that the emotional reaction probably was affected by the invasion of the participants' personal space, which also applies to virtual social interaction [39,63].

Further, based on the interpretation that virtual social touch shares influence factors with its unmediated counterpart, there are numerous potentially confounding factors that we did not control for in this specific study, but that influence social interaction in the real world. These include implicit social biases concerning individual assumptions about others and stereotypes towards in-group members and against out-group members $[17,35]$. We assume that such biases and aspects related to the perceivable traits of the interaction partner (e.g., the body height and perceived attractiveness of the interaction partner) contribute to the large inter-individual differences in the emotional evaluation we found. However, this assumption is still in line with our general interpretation. That is, virtual social touch can evoke different emotional responses moderated by factors that do also moderate the perception of unmediated social interaction.

Another limitation relates to the limited avatar individualization possibilities that did not allow participants to create a precise virtual representation. Thus, based on the Proteus effect [64] the avatar's identity cues that are not shared with the user's visual appearance that embodies it may have affected the touching behavior, attitudes, and experiences. For example, the perceived attractiveness of their avatar may have affected their acceptance of intimate behavior [64]. This potential confounding effect indicates that virtual social touch is also affected by effects exclusive to virtual interaction.

Given these limitations, we highlight, that our results regarding the valence and intensity of emotional responses are not readily transferable to any social interaction context or user constellation in virtual worlds beyond the characteristics of our study (i.e., prescribed touches, virtual interaction between heterosexual, opposite-sex strangers represented by realistic avatars with limited non-verbal communication cues, the interaction between avatars of white skin-tone, citizens of Central Europe). 


\subsection{Future Work}

The limitations above translate directly into follow up research questions and study designs. For example, a nuanced comparison of specific touch interactions regarding their impact on users should lead to more specific design recommendations for VR content creators, by answering what touch-types may be adequate for specific social mechanics. Thereby, we suggest considering more diverse constellations of participant and avatar characteristics in follow up studies. This would aid content creators to understand under which conditions specific social norms from the physical world are relevant in the virtual realm and when virtual interaction underlies its own rules (e.g., the impact of the sexual orientation of participants, implicit social biases, the quality of the interpersonal relationship, different cultural backgrounds, Proteus effect).

Another question which emerges from our sample characteristics relates to the impact of potential familiarization effects, i.e., does prior VR experiences affect the perception of virtual social body contact? We consider field studies within the current social VR platforms (online questionnaires or interviews with users) as an appropriate method to answer this question. This approach could also be valuable to evaluate social touch that occurs during spontaneous virtual interaction.

Concerning the Proteus effect, we note that some social VR applications feature unlimited avatar customization options (e.g., nonhumanoid avatars, excessively large or small avatars). Consequently, we consider the investigation of how appearance characteristics that do not apply to humans and social interaction outside of VR affect the experience of virtual social touch as an exciting field of research. In that sense, we currently focus on fostering desired and inhibit undesired experiences of virtual body contact based on variations of immersive characteristics of the interaction. Inspired by the idea to augment the social interaction in VR [45], we currently prepare a study on visual augmentations of virtual touches (e.g., particle effects on body contact) to manipulate emotional reactions.

\subsection{Practical Implications}

Our results inform practitioners about how they may implement mechanics related to virtual body contact in social VR. Our findings suggest that virtual social touch should be a moderated feature to induce desired but simultaneously limit negative emotional experiences during spontaneous encounters between strangers in social VR. Our results related to the negative emotions emphasize that virtual physical harassment [5] can have real emotional consequences. The large interindividual differences imply that there could always be individuals who are particularly sensitive to experiences in VR. Thus, developers should provide the user with adjustable privacy settings related to social touch capabilities. It is already common practice in social VR applications that users can configure a personal space bubble that prevents others from invading one's personal space. We think a natural extension of such a system would allow users to configure what types of touches they may allow with other users in specific contexts. Such a system could also be pre-configured automatically based on intraindividual personality characteristics (e.g., comfort with interpersonal touch).

Another value of the present results relates to the design of social features tied to virtual social touch. As an example, friending in
Recroom can be triggered by a handshake, what we would consider as an adequate interaction metaphor. In contrast, touching another avatar's pubic area for 20 seconds to send a friend request probably would be entirely inappropriate. This intentionally exaggerated example should clarify that the use of certain touch interactions for social VR features should always correspond to the social feature's underlying meaning. At the very least, the design of such interactions should always consider how these interactions are evaluated outside of VR and that they should not be mandatory for users to interact in the virtual world.

\section{CONCLUSION}

We investigated virtual social touch in a two-user VR scenario and found that it affects diverse emotions. Our results indicate that corresponding to unmediated social touch, touch intimacy, the direction of touch, and participants' sex modulate positive and negative affective responses to virtual touch. Thus, VR can induce meaningful interaction based on interpersonal touch, which resembles its unmediated counterpart in terms of applied norms and affective responses, even without realistic haptic feedback. To inform future research and practitioners, we indicated practical approaches for follow up studies and derived implications for the use of interpersonal touch in social VR applications. Eventually, a precise understanding of the aspects that shape our experience of virtual social interaction not only opens up the possibility to bring compelling emotional experiences to people who are physically separated from each other. It also enables us to develop mechanics that foster desired and prevent undesirable experiences induced by virtual social interactions.

\section{ACKNOWLEDGMENTS}

This work was partly supported by the European Regional Development Fund 2014-2020 in context of the project "MUVER" (EFRE0801066).

\section{REFERENCES}

[1] Imtiaj Ahmed, Ville Harjunen, Giulio Jacucci, Eve Hoggan, Niklas Ravaja, and Michiel M. Spapé. 2016. Reach out and Touch Me: Effects of Four Distinct Haptic Technologies on Affective Touch in Virtual Reality. In Proceedings of the 18th ACM International Conference on Multimodal Interaction (Tokyo, Japan) (ICMI '16). Association for Computing Machinery, New York, NY, USA, 341-348. https://doi.org/10.1145/2993148.2993171

[2] Jeremy N Bailenson and Nick Yee. 2008. Virtual interpersonal touch: Haptic interaction and copresence in collaborative virtual environments. Multimedia Tools and Applications 37, 1 (2008), 5-14.

[3] Christopher C Berger, Mar Gonzalez-Franco, Eyal Ofek, and Ken Hinckley. 2018. The uncanny valley of haptics. Science Robotics 3, 17 (2018), Art-No.

[4] Malin Björnsdotter and Håkan Olausson. 2011. Vicarious responses to social touch in posterior insular cortex are tuned to pleasant caressing speeds. fournal of Neuroscience 31, 26 (2011), 9554-9562.

[5] L. Blackwell, N. Ellison, N. Elliott-Deflo, and R. Schwartz. 2019. Harassment in Social VR: Implications for Design. In 2019 IEEE Conference on Virtual Reality and $3 D$ User Interfaces (VR). 854-855.

[6] S-J Blakemore, Davina Bristow, Geoffrey Bird, Chris Frith, and James Ward. 2005. Somatosensory activations during the observation of touch and a case of vision-touch synaesthesia. Brain 128, 7 (2005), 1571-1583.

[7] Pierre Bourdin, Josep Maria Tomàs Sanahuja, Carlota Crusafon Moya, Patrick Haggard, and Mel Slater. 2013. Persuading People in a Remote Destination to Sing by Beaming There. In Proceedings of the 19th ACM Symposium on Virtual Reality Software and Technology (Singapore) (VRST '13). ACM, New York, NY, USA, 123-132. https://doi.org/10.1145/2503713.2503724

[8] Carissa J Cascio, David Moore, and Francis McGlone. 2019. Social touch and human development. Developmental Cognitive Neuroscience 35 (2019), 5-11. 
[9] Inrak Choi, Eyal Ofek, Hrvoje Benko, Mike Sinclair, and Christian Holz. 2018 Claw: A multifunctional handheld haptic controller for grasping, touching, and triggering in virtual reality. In Proceedings of the 2018 CHI Conference on Human Factors in Computing Systems. ACM, 654.

[10] Dan-Mikael Ellingsen, Johan Wessberg, Olga Chelnokova, Håkan Olausson, Bruno Laeng, and Siri Leknes. 2014. In touch with your emotions: oxytocin and touch change social impressions while others' facial expressions can alter touch. Psychoneuroendocrinology 39 (2014), 11-20.

[11] Jos Feys. 2016. Nonparametric tests for the interaction in two-way factorial designs using R. The R fournal 8, 1 (2016), 367-378.

[12] Tiffany Field. 2002. Infants' need for touch. Human Development 45, 2 (2002), 100

[13] Kory Floyd. 2000. Affectionate same-sex touch: The influence of homophobia on observers' perceptions. The fournal of social psychology 140, 6 (2000), 774-788.

[14] Martina Fusaro, Matteo Lisi, Gaetano Tieri, and Salvatore Aglioti. 2020. Touched by vision: how heterosexual, gay, and lesbian people react to the view of their avatar being caressed on taboo body parts (pre-print). https://doi.org/10.31234/osf.io/dkzj5

[15] Alberto Gallace and Charles Spence. 2010. The science of interpersonal touch an overview. Neuroscience \& Biobehavioral Reviews 34, 2 (2010), 246-259.

[16] Mar Gonzalez-Franco and Tabitha C Peck. 2018. Avatar embodiment. towards a standardized questionnaire. Frontiers in Robotics and AI 5 (2018), 74.

[17] Anthony G Greenwald and Mahzarin R Banaji. 1995. Implicit social cognition: attitudes, self-esteem, and stereotypes. Psychological review 102, 1 (1995), 4

[18] Antal Haans and Wijnand IJsselsteijn. 2006. Mediated social touch: a review of current research and future directions. Virtual Reality 9, 2-3 (2006), 149-159.

[19] Judith A Hall and Ellen M Veccia. 1990. More" touching" observations: New insights on men, women, and interpersonal touch. Fournal of personality and social psychology 59, 6 (1990), 1155.

[20] Ville Johannes Harjunen, Michiel Spapé, Imtiaj Ahmed, Giulio Jacucci, and N Ravaja. 2017. Individual differences in affective touch: Behavioral inhibition and gender define how an interpersonal touch is perceived. Personality and Individual Differences 107 (2017), 88-95.

[21] Ville Johannes Harjunen, Michiel Spapé, Imtiaj Ahmed, Giulio Jacucci, and Niklas Ravaja. 2018. Persuaded by the machine: The effect of virtual nonverbal cues and individual differences on compliance in economic bargaining. Computers in Human Behavior 87 (2018), 384-394.

[22] Cindy Harmon-Jones, Brock Bastian, and Eddie Harmon-Jones. 2016. The discrete emotions questionnaire: A new tool for measuring state self-reported emotions. PloS one 11, 8 (2016), e0159915.

[23] Chad Harms and Frank Biocca. 2004. Internal consistency and reliability of the networked minds measure of social presence. (2004).

[24] Matthew J Hertenstein, Julie M Verkamp, Alyssa M Kerestes, and Rachel M Holmes. 2006. The communicative functions of touch in humans, nonhuman primates, and rats: a review and synthesis of the empirical research. Genetic, social, and general psychology monographs 132, 1 (2006), 5-94.

[25] Richard Heslin, Tuan D Nguyen, and Michele L Nguyen. 1983. Meaning of touch The case of touch from a stranger or same sex person. Fournal of Nonverbal Behavior 7, 3 (1983), 147-157.

[26] Matthias Hoppe, Beat Rossmy, Daniel Peter Neumann, Stephan Streuber, Albrecht Schmidt, and Tonja-Katrin Machulla. 2020. A Human Touch: Social Touch Increases the Perceived Human-likeness of Agents in Virtual Reality. In Proceedings of the 2020 CHI Conference on Human Factors in Computing Systems. 1-11.

[27] Gijs Huisman. 2017. Social touch technology: A survey of haptic technology for social touch. IEEE transactions on haptics 10, 3 (2017), 391-408.

[28] Wijnand A IJsselsteijn, Yvonne A W de Kort, and Antal Haans. 2006. Is this my hand I see before me? The rubber hand illusion in reality, virtual reality, and mixed reality. Presence: Teleoperators and Virtual Environments 15, 4 (2006), 455-464.

[29] Marcel Jonas, Steven Said, Daniel Yu, Chris Aiello, Nicholas Furlo, and Douglas Zytko. 2019. Towards a Taxonomy of Social VR Application Design. In Extended Abstracts of the Annual Symposium on Computer-Human Interaction in Play Companion Extended Abstracts (Barcelona, Spain) (CHI PLAY'19 Extended Abstracts). Association for Computing Machinery, New York, NY, USA, 437-444. https://doi.org/10.1145/3341215.3356271

[30] Stanley E Jones and A Elaine Yarbrough. 1985. A naturalistic study of the meanings of touch. Communications Monographs 52, 1 (1985), 19-56.

[31] Todd B Kashdan, James Doorley, Melissa C Stiksma, and Matthew J Hertenstein 2017. Discomfort and avoidance of touch: new insights on the emotional deficits of social anxiety. Cognition and Emotion 31, 8 (2017), 1638-1646.

[32] Anouk Keizer, Jutta R de Jong, Lianne Bartlema, and Chris Dijkerman. 2019. Visual perception of the arm manipulates the experienced pleasantness of touch Developmental cognitive neuroscience 35 (2019), 104-108.

[33] Robert S Kennedy, Norman E Lane, Kevin S Berbaum, and Michael G Lilienthal. 1993. Simulator sickness questionnaire: An enhanced method for quantifying simulator sickness. The international journal of aviation psychology 3, 3 (1993), 203-220.

[34] Konstantina Kilteni, Raphaela Groten, and Mel Slater. 2012. The sense of embodiment in virtual reality. Presence: Teleoperators and Virtual Environments 21, 4
(2012), 373-387.

[35] Jennifer T Kubota, Mahzarin R Banaji, and Elizabeth A Phelps. 2012. The neuroscience of race. Nature neuroscience 15, 7 (2012), 940-948.

[36] Anatole Lécuyer. 2009. Simulating haptic feedback using vision: A survey of research and applications of pseudo-haptic feedback. Presence: Teleoperators and Virtual Environments 18, 1 (2009), 39-53.

[37] Anatole Lecuyer, Sabine Coquillart, Abderrahmane Kheddar, Paul Richard, and Philippe Coiffet. 2000. Pseudo-haptic feedback: can isometric input devices simulate force feedback?. In Proceedings IEEE Virtual Reality 2000 (Cat. No. 00CB37048). IEEE, 83-90.

[38] Jaeyeon Lee, Mike Sinclair, Mar Gonzalez-Franco, Eyal Ofek, and Christian Holz. 2019. TORC: A Virtual Reality Controller for In-Hand High-Dexterity Finger Interaction. In Proceedings of the 2019 CHI Conference on Human Factors in Computing Systems. ACM, 71.

[39] Joan Llobera, Bernhard Spanlang, Giulio Ruffini, and Mel Slater. 2010. Proxemics with multiple dynamic characters in an immersive virtual environment. $A C M$ Transactions on Applied Perception (TAP) 8, 1 (2010), 1-12.

[40] Antonella Maselli and Mel Slater. 2013. The building blocks of the full body ownership illusion. Frontiers in human neuroscience 7 (2013), 83

[41] Ciara McCabe, Edmund T Rolls, Amy Bilderbeck, and Francis McGlone. 2008. Cognitive influences on the affective representation of touch and the sight of touch in the human brain. Social cognitive and affective neuroscience 3, 2 (2008), 97-108.

[42] Kimihiro Noguchi, Yulia R Gel, Edgar Brunner, and Frank Konietschke. 2012. nparLD: an R software package for the nonparametric analysis of longitudinal data in factorial experiments. Fournal of Statistical Software 50, 12 (2012).

[43] Valeria I Petkova and H Henrik Ehrsson. 2008. If I were you: perceptual illusion of body swapping. PloS one 3, 12 (2008).

[44] Michael Rietzler, Florian Geiselhart, Julian Frommel, and Enrico Rukzio. 2018. Conveying the perception of kinesthetic feedback in virtual reality using stateof-the-art hardware. In Proceedings of the 2018 CHI Conference on Human Factors in Computing Systems. ACM, 460.

[45] Daniel Roth, Gary Bente, Peter Kullmann, David Mal, Chris Felix Purps, Kai Vogeley, and Marc Erich Latoschik. 2019. Technologies for social augmentations in user-embodied virtual reality. In 25th ACM Symposium on Virtual Reality Software and Technology. 1-12.

[46] Valentina Russo, Cristina Ottaviani, and Grazia Fernanda Spitoni. 2020. Affective touch: A meta-analysis on sex differences. Neuroscience \& Biobehavioral Reviews 108 (2020), 445-452.

[47] Nathan Schmulewitz, Sudip K Ghosh, and Judy W Bowers. 2009. T1091 Pilot Study Examining Role of Healing Touch As An Adjunct to Conscious Sedation During EUS Procedures. Gastroenterology 136, 5 (2009), A-497.

[48] Thomas Schubert, Frank Friedmann, and Holger Regenbrecht. [n.d.]. igroup presence questionnaire (IPQ) overview. http://www.igroup.org/pq/ipq/index.php. [Online; accessed 24-September-2019].

[49] Mel Slater, Daniel Pérez Marcos, Henrik Ehrsson, and Maria V Sanchez-Vives. 2008. Towards a digital body: the virtual arm illusion. Frontiers in human neuroscience 2 (2008), 6

[50] Mel Slater, Bernhard Spanlang, Maria V Sanchez-Vives, and Olaf Blanke. 2010. First person experience of body transfer in virtual reality. PloS one 5, 5 (2010).

[51] Lapp Hanna Sophie and Croy Ilona. 2020. Insights from the German Version of the Social Touch Questionnaire: How Attitude towards Social Touch relates to Symptoms of Social Anxiety. Neuroscience (2020).

[52] Hidenobu Sumioka, Aya Nakae, Ryota Kanai, and Hiroshi Ishiguro. 2013. Huggable communication medium decreases cortisol levels. Scientific reports 3 (2013), 3034.

[53] Juulia T Suvilehto, Enrico Glerean, Robin IM Dunbar, Riitta Hari, and Lauri Nummenmaa. 2015. Topography of social touching depends on emotional bonds between humans. Proceedings of the National Academy of Sciences 112, 45 (2015), 13811-13816.

[54] Juulia T Suvilehto, Lauri Nummenmaa, Tokiko Harada, Robin IM Dunbar, Riitta Hari, Robert Turner, Norihiro Sadato, and Ryo Kitada. 2019. Cross-cultural similarity in relationship-specific social touching. Proceedings of the Royal Society B 286, 1901 (2019), 20190467.

[55] Justyna Swidrak and Grzegorz Pochwatko. 2019. Being Touched by a Virtual Human.: Relationships Between Heart Rate, Gender, Social Status, and Compliance. In Proceedings of the 19th ACM International Conference on Intelligent Virtual Agents. ACM, 49-55.

[56] Theresa Jean Tanenbaum, Nazely Hartoonian, and Jeffrey Bryan. 2020. " How do I make this thing smile?" An Inventory of Expressive Nonverbal Communication in Commercial Social Virtual Reality Platforms. In Proceedings of the $2020 \mathrm{CHI}$ Conference on Human Factors in Computing Systems. 1-13.

[57] Stephen Thayer. 1986. History and strategies of research on social touch. fournal of Nonverbal Behavior 10, 1 (1986), 12-28.

[58] Mark Tomita. 2008. Exploratory Study of Touch zones in college students on two campuses. Californian fournal of Health Promotion 6, 1 (2008), 1-22.

[59] Line Tremblay, Mélina Roy-Vaillancourt, Brahim Chebbi, Stéphane Bouchard, Michael Daoust, Jessica Dénommée, and Moriah Thorpe. 2016. Body image and 
anti-fat attitudes: an experimental study using a haptic virtual reality environment to replicate human touch. Cyberpsychology, Behavior, and Social Networking 19, 2 (2016), 100-106.

[60] Jan BF Van Erp and Alexander Toet. 2015. Social touch in human-computer interaction. Frontiers in digital humanities 2 (2015), 2.

[61] Thomas Waltemate, Dominik Gall, Daniel Roth, Mario Botsch, and Marc Erich Latoschik. 2018. The impact of avatar personalization and immersion on virtual body ownership, presence, and emotional response. IEEE transactions on visualization and computer graphics 24, 4 (2018), 1643-1652.

[62] Andrea Webb and Joann Peck. 2015. Individual differences in interpersonal touch On the development, validation, and use of the "comfort with interpersonal
touch"(CIT) scale. Journal of consumer psychology 25, 1 (2015), 60-77.

[63] Laurie M Wilcox, Robert S Allison, Samuel Elfassy, and Cynthia Grelik. 2006. Personal space in virtual reality. ACM Transactions on Applied Perception (TAP) 3 , 4 (2006), 412-428.

[64] Nick Yee and Jeremy Bailenson. 2007. The Proteus effect: The effect of transformed self-representation on behavior. Human communication research 33, 3 (2007), 271-290.

[65] Yuguang Zhao, Jaap Ham, and Jurgen van der Vlist. 2017. Persuasive virtual touch: The effect of artificial social touch on shopping behavior in virtual reality. In International Workshop on Symbiotic Interaction. Springer, 98-109. 